\title{
Aspectos diferenciales del riesgo de abandono al inicio del tratamiento de la adicción a la cocaína en pacientes con trastornos de la personalidad
}

\section{Differential aspects of treatment dropout risk in cocaine dependent patients with and without personality disorders}

\author{
José Miguel Martínez-González*, Natalia Albein-Urios**, Oscar Lozano-Rojas*** y Antonio \\ VERDEJO-GARCÍA**, **** \\ *Centro Provincial de Drogodependencias de Granada. España. ** Departamento de Personalidad, evaluación y tratamien- \\ to psicológico. Facultad de Psicología. Universidad de Granada. España. *** Facultad de Psicología. Universidad de Huelva. \\ España. **** Escuela de Psicología y Psiquiatría. Monash University. Melbourne, Australia.
}

\section{Resumen}

El objetivo de este trabajo fue conocer el riesgo de abandono del tratamiento de pacientes en tratamiento por su adicción a la cocaína en función de variables como la psicopatología en el Eje II, el nivel de gravedad del ajuste psicológico o la impulsividad. La muestra estaba compuesta por 95 pacientes, de los que el $53.7 \%$ presentaba un trastorno de la personalidad. Se utilizó una metodología descriptiva que nos permitió conocer las correlaciones entre diferentes variables, su capacidad predictiva y las diferencias entre grupos con respecto a variables relacionadas con el abandono del tratamiento. Este trabajo muestra que el riesgo de abandono durante los dos primeros meses no viene determinado por la presencia de un trastorno de la personalidad. En relación a los perfiles cognitivos de personalidad se comprueba que el antisocial presenta más riesgo de abandono. El perfil de paciente con más riesgo de abandono del tratamiento al iniciar el tratamiento presenta un menor nivel de calidad de vida autopercibida y niveles más elevados de impulsividad, en especial la experiencia de fuertes impulsos bajo condiciones de afectos positivos. Se ha comprobado que la presencia de sentimientos adversos como afectos, emociones, sensaciones y sentimientos que afectan al paciente es la dimensión que tiene un papel más destacado en el análisis del riesgo de abandono del tratamiento. Este trabajo permite poner de relieve las variables que deben atenderse con más especificidad desde el inicio del tratamiento porque se han relacionado más claramente con un mayor riesgo de abandono del tratamiento.

Palabras Clave: abandono, adherencia, trastornos de la personalidad, tratamiento, cocaína.

\begin{abstract}
The aim of this study was to examine the link between psychosocial adjustment, impulsivity and comorbid Axis II psychopathology, and the risk of treatment dropout in cocaine dependent patients. The sample consisted of 95 cocaine dependent participants, $53.7 \%$ of whom had been diagnosed with Axis II personality disorders. We utilised a descriptive methodology, which allowed us to examine correlations between the different variables, group differences in these variables, and their ability to predict different dimensions associated with the risk of dropout. Results show that the risk of dropout during the first two months of treatment is not associated with the existence of comorbid personality disorders. With regard to the cognitive profiles of personality disorders, we show that the antisocial profile is associated with higher risk of dropout. The profile of patients at higher risk of treatment dropout is characterised by lower levels of perceived quality of life and elevated levels of impulsivity, especially the experience of strong impulses under positive affect. Therefore, the presence of psychological dimensions tightly associated with emotions, affects and subjective feelings are the more relevant to estimate the risk of treatment dropout in cocaine dependent patients. This study stresses the variables that need to be specifically addressed during early phases of cocaine addiction treatment, since they are associated with higher risk of treatment dropout.

Key Words: treatment dropout, compliance, personality disorders, treatment, cocaine.
\end{abstract}


$\mathrm{E}$ 1 abandono prematuro del tratamiento es frecuente entre los pacientes cocainómanos (Caballero, 2005). Estudios como el de Higgins et al. (1994), encuentran que hasta un $80 \%$ de estos pacientes abandonan el tratamiento y al parecer la mayoría de ellos lo hacen durante los tres primeros meses (Sánchez-Hervás et al., 2010). Estudios sobre la psicoterapia de los trastornos de la personalidad (TP) han encontrado por un lado que entre un 10 y un $30 \%$ de ellos abandonan el tratamiento (Perry y Bond, 2002), teniendo en cuenta, no obstante que en general existe un índice bajo de abandonos una vez que el paciente ha iniciado la terapia (Tyrer y Davidson, 2002).

En el tratamiento de las drogodependencias se ha constatado que la permanencia de los pacientes en el mismo es un predictor significativo de su éxito, porque una buena adherencia al tratamiento disminuye la probabilidad de abandono (Caballero, 1993; Jackson, 2002; NIDA, 2002; Pretzer, 1998). En la alianza terapéutica entre paciente y terapeuta, que como se sabe es un pilar clave de la adherencia al tratamiento, se pueden diferenciar tres elementos: el vínculo entre paciente y terapeuta, la tarea que se propone como motor para el cambio y los objetivos del tratamiento (Bordin, 1979). Si bien la motivación al cambio es un factor significativo en el cumplimiento de sesiones y plan de recuperación individual (Daley y Zuckoff, 2004), esta podría estar influida por la presencia de TP, siendo por tanto éste un posible nexo entre esta psicopatología y el abandono del tratamiento. Un meta-análisis sobre la adherencia encontró que la presencia de TP se relaciona con mal cumplimiento de las indicaciones terapéuticas, tasas más bajas de adherencia y fracaso terapéutico (Sirvent, 2009). En otros, sin embargo, no se encontró un vínculo directo entre TP y abandono del tratamiento (Verheul, Van den Bosch y Ball, 2007). No obstante, la presencia de TP puede tener cierto peso en la evolución del tratamiento porque la adherencia al tratamiento decrece cuando el diagnóstico de TP pesa más que la calidad de la relación terapéutica (Hersoug, Monsen, Havik y Oglend, 2002).

Se ha visto que la presencia de un TP no determina por sí mismo el resultado del tratamiento (Fernández, López y Becoña, 2010; Martínez-González, Graña y Trujillo, 2009) de modo que lo que realmente es significativo en la construcción de la adherencia al tratamiento, y por tanto en la permanencia de los pacientes en tratamiento, es la calidad de relación terapéutica.

Queda por determinar el papel del malestar emocional en la adherencia al tratamiento porque algunos estudios han mostrado la importancia que puede llegar a tener la presencia de trastornos del estado de ánimo. Al parecer influye negativamente en la adherencia al elevar el riesgo de inconformidad del paciente con el tratamiento y como consecuencia incrementando la posibilidad de que abandone prematuramente (Kokkevi, Stefanis, Anastasopolou y Kostogianni, 1998). Igualmente sucede con niveles eleva- dos de distrés (Sánchez-Hervas, Tomás, Molina, Del Olmo y Morales, 2002), aunque también se ha visto que la ansiedad podría predecir una mayor retención en el tratamiento (Kokkevi et al., 1998).

Otra variable muy influyente en la adherencia es la impulsividad. El nivel de impulsividad se relaciona con una adherencia al tratamiento deficiente y disponemos de datos que muestran que los consumidores de cocaína tienen más problemas en el control de impulsos. Si además estos presentan un TP narcisista, límite o antisocial, se sabe que la impulsividad tarda más en disminuir una vez que el paciente ha dejado de consumir en comparación a lo que sucede con pacientes que no presentan esta psicopatología (Forcada, Pardo y Bondía, 2006). De esto se desprende que los pacientes que presentan una impulsividad elevada durante más tiempo permanecen durante más tiempo en una situación de mayor riesgo de abandono del tratamiento.

Las sesiones iniciales de tratamiento constituyen un elemento clave para la continuidad del tratamiento porque influyen significativamente en la imagen que los pacientes tienen del modelo terapéutico que se les ofrece. Por este motivo, las primeras sesiones deberían promover la confianza y percepción de utilidad del tratamiento (Long, Williams, y Hollin, 1999). Tal es así que se ha comprobado que la adherencia al tratamiento viene determinada por la propia recepción o acogida dispensada al paciente a su llegada al dispositivo asistencial (Pedrero y Puerta, 2001). Cuando se establece con prontitud una buena alianza terapéutica, el abandono, si se produce, es más tardío. Esto muestra que las primeras sesiones y en general el comienzo de tratamiento tiene un peso significativo en el posterior desarrollo de la terapia. Incluso, se ha constatado que a lo largo de las primeras sesiones se producen logros muy significativos en la recuperación del paciente (Lambert, Hansem y Finch, 2001). En definitiva, se sabe que la alianza representa un porcentaje de varianza explicada del resultado final del tratamiento (Lingiardi, Filippucci y Baiocoo, 2005).

Los objetivos del estudio fueron: conocer el perfil de un grupo de pacientes con diagnóstico de dependencia de cocaína que iniciaban tratamiento con respecto al riesgo de abandono e identificar las variables que pudieran estar relacionadas con el riesgo de abandono del tratamiento como la presencia de TP, el ajuste psicológico, los perfiles de personalidad, la impulsividad y la calidad de vida (CV).

\section{Método}

\section{Participantes}

La muestra la componen 95 pacientes con trastorno por dependencia a la cocaína, que inician tratamiento en el Centro Provincial de Drogodependencias de Granada. Debían ser pacientes que se encontraran en los primeros 45 días del tratamiento y hubiesen logrado estar abstinentes al menos 20 días en el momento de la evaluación. La media de edad 
de los participante fue de 32.66 años en un rango de edad de 19 a 52 años. El $87.4 \%$ eran hombres $(\mathrm{n}=83)$ mientras que el $12.6 \%$ eran mujeres $(n=12)$. Presentaban un TP el $53.7 \%(\mathrm{n}=51)$ de la muestra, siendo la distribución de TP la siguiente: antisocial $6.3 \%(n=4)$, límite $16.8 \%(n=16)$, histriónico $11.6 \%(\mathrm{n}=11)$, narcisista $1.1 \%(\mathrm{n}=1)$, evitativo $11.6 \%(\mathrm{n}=11)$, obsesivo-compulsivo $6.3 \%(\mathrm{n}=6)$.

A todos los pacientes se les solicitó el consentimiento informado.

\section{Instrumentos}

La valoración del nivel de riesgo de abandono del tratamiento se hizo mediante el cuestionario VAP-30 (Sirvent, 2010). Se diseñó para identificar en las primeras sesiones los pacientes más adherentes determinando el nivel de riesgo de abandono del tratamiento en los dos meses siguientes. Consta de 30 ítems con una escala general de 14 ítems y 5 subescalas o factores que recogen variables de adherencia terapéutica: motivación, conciencia de problema, sentimientos adversos, tratamiento y adherencia. La "escala general" proporciona un índice de riesgo de abandono del tratamiento por parte del paciente; la "motivación" se refiere a las ganas de recuperarse y llevar a cabo el tratamiento activamente con las renuncias y limitaciones que ello comporta; la "conciencia de problema" que representa el grado de conocimiento y capacidad del paciente para objetivar la drogadicción con las renuncias y limitaciones que eso lleva consigo; los "sentimientos adversos" que son aquellos afectos, sensaciones y sentimientos que afectan con mayor frecuencia al sujeto; los "deseos de consumo" son el anhelo o ansia por consumir drogas y/o por el ambiente toxicofílico; el factor "tratamiento" traduce la valoración subjetiva del programa terapéutico en cuanto a la confianza, dificultad, adecuación y aceptación del mismo por parte del sujeto; la "adherencia" indica que el sujeto no va a abandonar el tratamiento, no su eficaz cumplimiento. En todas las dimensiones, la mayor puntuación indica mayor riesgo de abandono.

Para indagar en los esquemas prototípicos de los diferentes perfiles de personalidad se utilizó el PBQ (Personality Beliefs Questionnaire). El Personality Belief Questionnaire (PBQ, Beck y Beck, 1991) es un cuestionario diseñado para medir el grado con el que una persona se identifica con creencias básicas disfuncionales relacionadas con los TP. El cuestionario consta de 126 ítems, incluyendo diez escalas correspondientes a cada uno de los trastornos del Eje II (DSMIV-TR, 2002). Nueve de las escalas están compuestas por 14 ítems. Las escalas son: evitativo, dependiente, obsesivo-compulsivo, narcisista, antisocial, histriónico, esquizoide y paranoide. Una escala adicional mide el trastorno límite de la personalidad, compuesta de 14 ítems extraídos del resto de escalas. Presenta respuestas tipo likert de cinco valores. El cuestionario se encuentra actualmente en fase de validación en lengua castellana (Albein-Urios, Martínez-González, Lozano-Rojas y Verdejo-García, 2011).
Para la evaluación de la psicopatología no psicótica se utilizó el Cuestionario General sobre Salud - General Health Questionnarie - GHQ-28 (Goldber y Hiller, 1979), el cual establece un índice de gravedad de ajuste psicológico construido a partir de la suma de cuatro subescalas: síntomas somáticos, ansiedad, disfunción social y depresión (González-Saiz et al., 1997).

Para la evaluación y diagnóstico de los TP se utilizó la entrevista semiestructurada "Examen Internacional de los Trastornos de la Personalidad (IPDE)" (Loranger et al. 1991; versión española de López-Ibor, Pérez Urdániz y Rubio, 1996).

La evaluación del Eje I se llevó a cabo a través de entrevista clínica, atendiendo a criterios DSM-IV-TR (APA, 2002). Para confirmar la presencia de psicopatología en el Eje, I se utilizó la SCID-I (First, Spitzer, Gibbon y Williams, 1999).

La impulsividad-rasgo se evaluó mediante el cuestionario UPPS (Whiteside y Lynam, 2001; Adaptación española: Verdejo-García, Lozano, Moya, Alcázar y Pérez-García, 2010). Consta de 59 ítems divididos en 5 subescalas: urgencia positiva, urgencia negativa, (tendencia a experimentar fuertes impulsos bajo condiciones de afecto negativo) falta de premeditación (tendencia a reflexionar sobre las consecuencias de un acto antes de que se precipite), falta de perseverancia (capacidad para permanecer centrado en una tarea que puede ser aburrida o difícil) y búsqueda de sensaciones (tendencia a disfrutar y realizar actividades emocionantes y apertura a experiencias novedosas que pueden o no ser peligrosas).

Para medir la CV se utilizó el cuestionario TECVASP que es un cuestionario específico para la evaluación de la CV en drogodependientes (Lozano, Rojas y Pérez, 2006). Consta de 20 ítems con formato de respuesta graduada con cinco categorías: nada, poco, a veces, bastante, mucho. Puntuaciones altas en el test indican buena CV relacionada con la salud y puntuaciones bajas es un indicador de una mala CV.

\section{Análisis estadístico}

El estudio de las relaciones entre las puntuaciones del VPA con otras variables con las que teóricamente deben existir relaciones se ha realizado a través de correlaciones de Pearson o chi $^{2}$, según la naturaleza métrica de las variables. Por otro lado, se ha realizado un análisis multivariado para conocer la capacidad explicativa de las variables que mostraron relaciones significativas sobre las puntuaciones del VPA. El análisis se hizo con el programa SPSS 19.0.

\section{Procedimiento}

La caracterización psicopatológica de la muestra la realizaron psicólogos del Centro Provincial de Drogodependencias de Granada y la evaluación de las variables neuropsicológicas y caracterización de la muestra una psicóloga miembro de nuestro grupo de investigación. El diagnóstico psicopatológico se realizó durante las sesiones de evaluación y la 
evaluación neuropsicológica mediante sesiones individuales adicionales al tratamiento. Algunos instrumentos como el cuestionario VPA-30 y PBQ los completaron los pacientes en sus hogares, entregándolos tras una semana desde que se realizó la evaluación neuropsicológica.

\section{Resultados}

El análisis de la CV y las dimensiones del cuestionario de adherencia muestra que una percepción de CV menor al inicio de tratamiento, se relaciona positiva y significativamente con un mayor riesgo de abandono centrado en variables como motivación para realizar el tratamiento, la presencia de sentimientos adversos y deseos de consumo (tabla 1).

En el análisis de la relación de las dimensiones del cuestionario VPA-30 con los factores del cuestionario de impulsividad, se aprecia que solo las urgencias, tanto positivas como negativas, presentan correlaciones positivas y estadísticamente significativas con algunas de las dimensiones indicadoras del riesgo de abandono del tratamiento. Las urgencias negativas correlacionan con mayores puntuaciones de riesgo en las dimensiones como sentimientos adversos y deseos de consumo, mientras que las urgencias positivas lo hacen además de las mencionadas, con el riesgo que viene dado por el nivel de conciencia de problema y valoración subjetiva del programa terapéutico en relación a su confianza en él, la dificultad que entraña para el paciente, su adecuación y aceptación (tabla 2). Por el contrario, no se observa que las dimensiones del cuestionario UPPS premeditación, perseverancia y búsqueda de sensaciones se relacionen con mayor riesgo de abandono.

No se han encontrado diferencias estadísticamente significativas en las dimensiones del cuestionario VPA-30 en función de la presencia de psicopatología en el Eje II, por

Tabla 1

Correlaciones de los factores del cuestionario de VPA, la calidad de vida y el ajuste psicológico

\begin{tabular}{|c|c|c|c|c|c|c|c|}
\hline & & $\begin{array}{c}\text { Puntuación } \\
\text { total } \\
\text { TECVASP }\end{array}$ & GHQ28A & GHQ28B & GHQ28C & GHQ28D & $\begin{array}{l}\text { GHQ28 } \\
\text { TOTAL }\end{array}$ \\
\hline \multirow[t]{3}{*}{$\begin{array}{l}\text { VPA. } \\
\text { Motivación }\end{array}$} & $\begin{array}{l}\text { Correlación } \\
\text { de Pearson }\end{array}$ &,- 249 &,- 011 &,- 032 & ,023 & ,033 & ,007 \\
\hline & Sig. (bilateral) & .045 & .927 & .784 & 840 & .779 & ,951 \\
\hline & N & 65 & 77 & 77 & 77 & 76 & 77 \\
\hline \multirow[t]{3}{*}{$\begin{array}{c}\text { VPA. } \\
\text { Conciencia de problema }\end{array}$} & $\begin{array}{l}\text { Correlación } \\
\text { de Pearson }\end{array}$ &,- 190 &,- 024 &,- 051 &,- 065 &,- 012 &,- 040 \\
\hline & Sig. (bilateral) & , 130 & .833 & .660 & .572 & .915 & ,732 \\
\hline & N & 65 & 77 & 77 & 77 & 76 & 77 \\
\hline \multirow[t]{3}{*}{$\begin{array}{c}\text { VPA. } \\
\text { Sentimientos adversos }\end{array}$} & $\begin{array}{l}\text { Correlación } \\
\text { de Pearson }\end{array}$ &,- 376 & ,127 & .127 &, 128 & , 145 & , 160 \\
\hline & Sig. (bilateral) & ,002 & ,272 & .270 & .269 & 211 & ,166 \\
\hline & N & 65 & 77 & 77 & 77 & 76 & 77 \\
\hline \multirow[t]{3}{*}{$\begin{array}{c}\text { VPA. } \\
\text { Deseos de consumo }\end{array}$} & $\begin{array}{l}\text { Correlación } \\
\text { de Pearson }\end{array}$ &,- 408 & ,070 &, 142 & 111 &, 126 &, 140 \\
\hline & Sig. (bilateral) &, 001 & .545 & .218 & ,338 & .276 & .224 \\
\hline & N & 65 & 77 & 77 & 77 & 76 & 77 \\
\hline \multirow[t]{3}{*}{ VPA Tratamiento } & $\begin{array}{l}\text { Correlación } \\
\text { de Pearson }\end{array}$ &,- 210 & .014 & .002 &,- 045 & .042 & 011 \\
\hline & Sig. (bilateral) & ,094 & .903 & .989 & .699 & .719 & .924 \\
\hline & N & 65 & 77 & 77 & 77 & 76 & 77 \\
\hline \multirow[t]{3}{*}{$\begin{array}{c}\text { VPA. } \\
\text { Escala general }\end{array}$} & $\begin{array}{l}\text { Correlación } \\
\text { de Pearson }\end{array}$ &,- 209 &,- 029 &,- 044 &,- 018 & 031 &,- 014 \\
\hline & Sig. (bilateral) & ,094 & .802 & ,702 & 879 & ,793 &, 902 \\
\hline & $\mathrm{N}$ & 65 & 77 & 77 & 77 & 76 & 77 \\
\hline
\end{tabular}


Tabla 2

Correlaciones entre las dimensiones del cuestionario VPA-30y UPPS

\begin{tabular}{|c|c|c|c|c|c|c|}
\hline & & $\begin{array}{l}\text { Urgencias } \\
\text { negativas }\end{array}$ & $\begin{array}{c}\text { Falta de } \\
\text { premeditación }\end{array}$ & $\begin{array}{c}\text { Falta de } \\
\text { perseverancia }\end{array}$ & $\begin{array}{l}\text { Búsqueda de } \\
\text { sensaciones }\end{array}$ & $\begin{array}{c}\text { Urgencias } \\
\text { positivas }\end{array}$ \\
\hline \multirow[t]{3}{*}{$\begin{array}{c}\text { VPA. } \\
\text { Motivación }\end{array}$} & $\begin{array}{l}\text { Correlación de } \\
\text { Pearson }\end{array}$ & .124 & .026 &,- 082 & ,117 & 193 \\
\hline & Sig. (bilateral) & ,286 & .823 & .482 & .315 & .094 \\
\hline & N & 76 & 76 & 76 & 76 & 76 \\
\hline \multirow{3}{*}{$\begin{array}{c}\text { VPA. } \\
\text { Conciencia de } \\
\text { problema }\end{array}$} & $\begin{array}{l}\text { Correlación de } \\
\text { Pearson }\end{array}$ & 171 & .064 &,- 074 & , 138 & ,247 \\
\hline & Sig. (bilateral) & 140 &, 584 & .524 &, 234 &, 032 \\
\hline & $\mathrm{N}$ & 76 & 76 & 76 & 76 & 76 \\
\hline \multirow{3}{*}{$\begin{array}{c}\text { VPA. } \\
\text { Sentimientos } \\
\text { adversos }\end{array}$} & $\begin{array}{l}\text { Correlación de } \\
\text { Pearson }\end{array}$ & ,271 & .049 & .012 & 081 & 293 \\
\hline & Sig. (bilateral) &, 018 & .674 & .920 & .488 & .010 \\
\hline & N & 76 & 76 & 76 & 76 & 76 \\
\hline \multirow{3}{*}{$\begin{array}{l}\text { VPA. } \\
\text { Deseos de } \\
\text { consumo }\end{array}$} & $\begin{array}{l}\text { Correlación de } \\
\text { Pearson }\end{array}$ &, 317 &, 078 & 053 & 131 & ,392 \\
\hline & Sig. (bilateral) & .005 & .505 & 651 & .258 & .000 \\
\hline & N & 76 & 76 & 76 & 76 & 76 \\
\hline \multirow[t]{3}{*}{$\begin{array}{l}\text { VPA. } \\
\text { Tratamiento }\end{array}$} & $\begin{array}{l}\text { Correlación de } \\
\text { Pearson }\end{array}$ & , 165 & ,053 &,- 101 & ,140 & ,243 \\
\hline & Sig. (bilateral) & , 155 & .646 & .385 & .228 & .034 \\
\hline & $\mathrm{N}$ & 76 & 76 & 76 & 76 & 76 \\
\hline \multirow[t]{3}{*}{$\begin{array}{c}\text { VPA. } \\
\text { Escala general }\end{array}$} & $\begin{array}{l}\text { Correlación de } \\
\text { Pearson }\end{array}$ & 119 & ,017 &,- 066 & 087 & 174 \\
\hline & Sig. (bilateral) & .306 & 882 & .573 & ,452 & 133 \\
\hline & $\mathrm{N}$ & 76 & 76 & 76 & 76 & 76 \\
\hline
\end{tabular}

lo que el riesgo de abandono a lo largo de los dos primeros meses de tratamiento no debería ser diferente en función de la presencia de un TP (tabla 3). El análisis de la relación de los diferentes perfiles de personalidad mediante el cuestionario PBQ con las dimensiones del cuestionario VPA-30 muestra que mientras que el trastorno antisocial de la personalidad correlaciona significativamente de forma positiva con todas las dimensiones del cuestionario, los perfiles de personalidad obsesivo-compulsivo, narcisista e histriónico no correlacionan con ninguna (tabla 4). Esto muestra que al iniciar el tratamiento el perfil de personalidad de mayor riesgo de abandono es el antisocial y la dimensión que más correlaciona con los diferentes perfiles de personalidad es la relacionada con los sentimientos adversos. El factor tratamiento del cuestionario VPA-30 correlaciona significativamente con los perfiles antisociales, paranoide y límite.
El nivel de ajuste psicológico no correlaciona significativamente con las dimensiones del cuestionario de riesgo de abandono.

El patrón de consumo, tomando como referencia los gramos de unidades de consumo de cánnabis y cocaína durante el último mes, tampoco correlaciona con ninguna de las dimensiones que recogen el nivel de riesgo de abandono.

De entre las variables que mostraron relaciones estadísticamente significativas, se aplicó un análisis multivariado utilizando como variable dependiente la puntuación total en la escala VPA. El procedimiento de inclusión de variables fue el de pasos sucesivos.

Los resultados obtenidos muestran un modelo que explica el $19 \%$ de la varianza $\left(\mathrm{F}_{(2,45)}=6,511 ; \mathrm{p}<0.01\right)$. La variable retenida en el modelo fue las puntuaciones del trastorno antisocial de la personalidad $(\beta=.368 ; \mathrm{p}=.008)$. 
Tabla 3

Comparación de los factores del cuestionario VPA en función de la presencia de trastornos de la personalidad

\begin{tabular}{|c|c|c|c|c|c|}
\hline Factores VPA & & Media & DT & $\mathbf{t}$ & $\mathbf{p}$ \\
\hline \multirow[t]{2}{*}{ Motivación } & Presenta TP & 2.67 & 1.06 & -1.003 & .320 \\
\hline & No presenta TP & 1.95 & 3.94 & & \\
\hline \multirow[t]{2}{*}{ Conciencia de problema } & Presenta TP & 2.66 & 2.08 & -1.150 & .255 \\
\hline & No presenta TP & 2.21 & .83 & & \\
\hline \multirow[t]{2}{*}{ Sentimientos adversos } & Presenta TP & 2.95 & 2.65 & -.962 & .340 \\
\hline & No presenta TP & 2.46 & 1.07 & & \\
\hline \multirow[t]{2}{*}{ Deseos de consumo } & Presenta TP & 3.00 & 2.21 & -.881 & .382 \\
\hline & No presenta TP & 2.62 & .99 & & \\
\hline \multirow[t]{2}{*}{ Tratamiento } & Presenta TP & 2.45 & 2.45 & -1.01 & .316 \\
\hline & No presenta TP & 1.98 & .94 & & \\
\hline \multirow[t]{2}{*}{ Escala General } & Presenta TP & 3.52 & 6.41 & -1.040 & .302 \\
\hline & No presenta TP & 2.33 & .83 & & \\
\hline
\end{tabular}

Tabla 4

Relación entre los factores del cuestionario VPA y los perfiles de personalidad

\begin{tabular}{|c|c|c|c|c|c|c|c|c|c|c|c|}
\hline & & $\begin{array}{l}\text { EVITA- } \\
\text { TIVO_- } \\
\text { PBO }\end{array}$ & $\begin{array}{l}\text { DEPEN- } \\
\text { DIENTE_- } \\
\text { PBQ }\end{array}$ & $\begin{array}{c}\text { ANTISO- } \\
\text { CIAL_- } \\
\text { PBQ }\end{array}$ & $\begin{array}{c}\text { OBSE- } \\
\text { COMP_- } \\
\text { PBQ }\end{array}$ & $\begin{array}{c}\text { NARCISIS- } \\
\text { TA } \\
\text { PBO }\end{array}$ & $\begin{array}{c}\text { PASIVO- } \\
\text { AGRES_- } \\
\text { PBQ }\end{array}$ & $\begin{array}{l}\text { HISTRIO- } \\
\text { NICO_- } \\
\text { PBO }\end{array}$ & $\begin{array}{l}\text { ESQUI- } \\
\text { ZOIDE_- } \\
\text { PBQ }\end{array}$ & $\begin{array}{c}\text { PARA- } \\
\text { NOIDE_- } \\
\text { PBQ }\end{array}$ & $\begin{array}{l}\text { LÍMITE_- } \\
\text { PBQ }\end{array}$ \\
\hline \multirow[t]{3}{*}{$\begin{array}{c}\text { VPA. } \\
\text { Motivación }\end{array}$} & $\begin{array}{l}\text { Correlación } \\
\text { de Pearson }\end{array}$ & ,208 & ,070 & ,252 &,- 045 & ,030 & , 145 & ,020 & 155 & ,213 &, 210 \\
\hline & $\begin{array}{c}\text { Sig. } \\
\text { (bilateral) }\end{array}$ & ,089 & .575 & ,038 & ,714 & 808 & ,240 & 870 & ,207 & 081 & ,085 \\
\hline & $\mathrm{N}$ & 68 & 67 & 68 & 68 & 68 & 67 & 68 & 68 & 68 & 68 \\
\hline \multirow{3}{*}{$\begin{array}{c}\text { VPA. } \\
\text { Conciencia } \\
\text { de problema }\end{array}$} & $\begin{array}{l}\text { Correlación } \\
\text { de Pearson }\end{array}$ & ,233 & ,095 & ,242 & , 106 & ,225 & .179 & ,015 & ,248 & , 184 & ,237 \\
\hline & $\begin{array}{c}\text { Sig. } \\
\text { (bilateral) }\end{array}$ & .056 & .443 & ,047 & ,388 & .066 & , 147 & ,905 & ,041 & 133 & ,052 \\
\hline & $\mathrm{N}$ & 68 & 67 & 68 & 68 & 68 & 67 & 68 & 68 & 68 & 68 \\
\hline \multirow{3}{*}{$\begin{array}{l}\text { VPA. } \\
\text { Sentimien- } \\
\text { tos adversos }\end{array}$} & $\begin{array}{l}\text { Correlación } \\
\text { de Pearson }\end{array}$ &, 358 & ,301 & ,317 & ,030 & ,099 & ,246 & ,093 & .152 & ,303 & ,346 \\
\hline & $\begin{array}{c}\text { Sig. } \\
\text { (bilateral) }\end{array}$ & .003 & 013 & ,008 & ,809 & .423 & ,045 & .450 & ,216 & 012 & ,004 \\
\hline & $\mathrm{N}$ & 68 & 67 & 68 & 68 & 68 & 67 & 68 & 68 & 68 & 68 \\
\hline \multirow{3}{*}{$\begin{array}{l}\text { VPA. } \\
\text { Deseos de } \\
\text { consumo }\end{array}$} & $\begin{array}{l}\text { Correlación } \\
\text { de Pearson }\end{array}$ & ,244 & ,259 & ,339 & .126 & ,054 & , 108 & .136 & .097 & , 210 & ,210 \\
\hline & $\begin{array}{c}\text { Sig. } \\
\text { (bilateral) }\end{array}$ & .045 & ,035 & ,005 & .307 & .664 & ,385 & .269 & .432 & ,085 & ,086 \\
\hline & $N$ & 68 & 67 & 68 & 68 & 68 & 67 & 68 & 68 & 68 & 68 \\
\hline \multirow[t]{3}{*}{$\begin{array}{l}\text { VPA. } \\
\text { Tratamiento }\end{array}$} & $\begin{array}{l}\text { Correlación } \\
\text { de Pearson }\end{array}$ & ,176 & , 199 & ,267 & ,074 & 232, & ,223 & 131 & ,229 & ,241 & ,244 \\
\hline & $\begin{array}{c}\text { Sig. } \\
\text { (bilateral) }\end{array}$ & ,150 & .107 & ,028 &, 548 & ,057 & .070 & ,287 & ,060 & ,048 & ,045 \\
\hline & $N$ & 68 & 67 & 68 & 68 & 68 & 67 & 68 & 68 & 68 & 68 \\
\hline \multirow{3}{*}{$\begin{array}{l}\text { VPA. } \\
\text { Escala } \\
\text { general }\end{array}$} & $\begin{array}{l}\text { Correlación } \\
\text { de Pearson }\end{array}$ &, 356 & ,275 & .364 & .109 & ,207 & .275 & .147 & .219 & ,307 & .363 \\
\hline & $\begin{array}{c}\text { Sig. } \\
\text { (bilateral) }\end{array}$ & ,003 & ,024 & ,002 &, 376 & .090 & .024 & ,233 & ,073 & 011 & ,002 \\
\hline & $\mathrm{N}$ & 68 & 67 & 68 & 68 & 68 & 67 & 68 & 68 & 68 & 68 \\
\hline
\end{tabular}

NOTA: VAP-30: riesgo de abandono. 


\section{Discusión}

Este trabajo ha permitido analizar detalladamente la relación del riesgo de abandono con variables tan relevantes como la CV, la impulsividad o los TP. En primer lugar se constata que la presencia de un TP no puede vincularse con un mayor riesgo de abandono en el momento de iniciar el tratamiento. Esto es, los pacientes cocainómanos no abandonarían el tratamiento solo por el hecho de presentar este tipo de psicopatología. Esto evidencia que la patología dual incrementa las dificultades asociadas al desarrollo de una intervención psicoterapéutica, pero no puede asumirse que los pacientes que presentan un TP tienen más probabilidad de abandonar el tratamiento a lo largo de los dos primeros meses en comparación al resto (Becoña et al., 2011).

El análisis de la relación entre los diferentes patrones de personalidad y las variables del cuestionario de adherencia muestra que el perfil que más riesgo de abandono del tratamiento durante los dos primeros meses presenta es el antisocial. Este perfil se relaciona significativamente con todas las dimensiones del cuestionario de adherencia. Por el contrario, los perfiles obsesivo, narcisista e histriónico no se relacionan significativamente con el riesgo de abandono. En la línea de lo anterior, estos resultados evidencian que la probabilidad de que fracase el tratamiento no depende de la presencia de psicopatología en el Eje II, dado que existen diferentes niveles de riesgo en cada uno de ellos, confirmando que no es posible relacionar claramente un perfil de personalidad con el abandono del tratamiento (McMahon, Kelley y Konzckanar, 1993). Aunque podría establecerse cierta asociación entre las recaídas y las escalas básicas de la personalidad (López et al., 2007) si se hace la necesaria distinción entre abandono y recaída, queda claro que no debe asociarse a la recaída sin más al abandono del tratamiento. Más bien podría asociarse parcialmente a diferentes patrones de personalidad como se ha constatado también en otros trabajos previos (López et al., 2007). Este riesgo diferencial en el tratamiento de la adicción a la cocaína solo debe asimilarse con la necesidad de llevar a cabo diferentes estrategias terapéuticas.

Los resultados muestran que cuando los pacientes al iniciar el tratamiento perciben menores niveles de CV tienen más dificultad para identificar la utilidad del tratamiento que se le propone, más anhelo por consumir nuevamente cocaína y más sentimientos adversos. Esto puede indicar que un mayor nivel de CV percibido disminuye el riesgo de abandono del tratamiento durante los dos primeros meses, convirtiendo a esta variable en un objeto de intervención de primera magnitud en el tratamiento (Martínez-González, Graña y Trujillo, 2010).

El hecho de que el nivel de ajuste psicológico no influya en las dimensiones del cuestionario de riesgo de abandono, indica al menos al iniciar el tratamiento que el estado emocional situacional no media significativamente en la probabilidad de abandono prematuro del tratamiento, por lo que la ansiedad o estados depresivos no deberían asociarse al abandono sin más. El hecho de no haber encontrado una relación destacada entre el riesgo de abandono y el estado emocional negativo, como indican otros trabajos que describían una relación directa entre la presencia de un trastorno depresivo y el abandono prematuro del tratamiento en cocainómanos (Caballero, 2005) puede deberse a que al iniciar el tratamiento y lograr la abstinencia los pacientes se encuentran bajo el efecto de relajación que les lleva a minimizar los problemas y dificultades (Iraurgi, Sanz y Martínez-Pampliega, 2004).

Se observa una vez más el vínculo entre impulsividad y adherencia, en la medida en que mayores niveles de impulsividad se asocian a un mayor riesgo de abandono (Forcada et al., 2006; Sargeant, Bornavolova, Trotman y Lejnez, 2012). El análisis multivariado nos ha permitido conocer la relación entre el perfil antisocial y el riesgo de abandono. Este vínculo es compatible con la clínica de este trastorno de la personalidad, confirmándose por tanto la mayor probabilidad de abandono del tratamiento de pacientes del cluster impulsivo. Por extensión podría decirse que la alianza terapéutica podría verse igualmente afectada por la presencia de niveles elevados de impulsividad, dado que las urgencias positivas se relacionan inversamente con una valoración favorable del paciente respecto al tratamiento. Esto sin duda tiene gran peso, al menos en parte, en la probabilidad de construir una buena alianza terapéutica (Bordin, 1979). De esto se desprende que la dificultad que puede asociarse en el tratamiento de los pacientes con TP podría depender del tipo de trastorno a la vez que se constata el papel de la impulsividad en el fracaso para establecer una buena adherencia. El TP que más problemas en este sentido suele presentar el iniciar el tratamiento y por tanto presenta mayor riesgo de abandono es el perfil antisocial (Sargeant et al., 2012). El tratamiento debe por tanto incorporar en las fases iniciales estrategias que se dirijan a la disminución de la impulsividad (Vergara-Moragues, González-Saiz, Lozano y Verdejo, 2013).

Con respecto a la relación particular entre las urgencias positivas y negativas medidas a través del cuestionario UPPS y el cuestionario VPA-30 se constata el impacto de la impulsividad en el riesgo de abandono del tratamiento. Esto podría mostrar el peso de las emociones en los procesos de recaída en paciente dependientes de cocaína (Epstein et al., 2009). En la medida en que se incrementa la impulsividad ante estados emocionales positivos se incrementa el riesgo de abandono en lo que se refiere a la comodidad del paciente en el tratamiento, su conciencia de trastorno adictivo, presente más sentimientos adversos y deseo de consumir. Los resultados son compatibles con la observación clínica en la que se constata que es frecuente encontrar a lo largo del tratamiento de deshabituación psicológica en cocainómanos que estados de euforia se conviertan en factores de riesgo de recaída en el consumo. 
El hecho de que no se encuentren relaciones significativas de dimensiones de la impulsividad como la premeditación, la perseverancia o búsqueda de sensaciones podría indicar que éstas tienen un papel más central en fases más avanzadas del tratamiento, mientras que el riesgo de abandono al inicio del tratamiento en dependientes a la cocaína podría depender más del control de respuestas impulsivas ante emociones positivas y negativas.

El hecho de que los patrones o perfiles antisocial, paranoide y límite correlacionen con los factores del cuestionario de riesgo de abandono del tratamiento, muestra que estos patrones podrían tener diferencialmente más dificultad para establecer una buena adherencia al tratamiento, dado que la convicción de que el tratamiento que se lleva a cabo es el adecuado constituye una pieza muy importante para establecer una buena adherencia al tratamiento (Urbanoski, Kelly, Hoeppner y Slaymaker, 2012).

A diferencia del estudio de Sánchez-Hervás et al. (2010) en el que se asociaba el riesgo de abandono con nivel de gravedad del consumo, en nuestro estudio no hemos podido demostrar esta conexión, de modo que no podemos afirmar que el riesgo de abandono dependa del patrón del consumo que el paciente tiene durante el mes previo al inicio de tratamiento, como medida indirecta del nivel de gravedad.

En este trabajo se ha podido ver que el riesgo asociado con la presencia de sentimientos adversos es la dimensión que correlaciona con más perfiles de personalidad lo que viene a confirmar que es la presencia de sentimientos adversos como afectos, emociones, sensaciones y sentimientos que afectan al paciente la dimensión que tiene un papel más destacado en el análisis del riesgo de abandono el tratamiento (Sirvent, 2010).

Si bien es cierto que la presencia de psicopatología se relaciona con mayor dificultad en el desarrollo del tratamiento, los resultados muestran que un mayor riesgo de abandono del tratamiento a lo largo de los dos primeros meses no vendría determinado por la presencia de TP si bien es el perfil antisocial el que se puede vincular con este riesgo de un modo más claro en comparación a otros. El paciente con más riesgo sería aquel que al iniciar el tratamiento informara de una CV autopercibida más baja, a la vez que niveles más elevados de impulsividad, en especial la referida a las urgencias positivas, esto es, aquellos que tienden a experimentar fuertes impulsos bajo condiciones de afectos positivos.

La evaluación inicial debería incorporar el análisis de las variables que parecen estar vinculadas con un mayor riesgo de abandono prematuro del tratamiento, porque la detección precoz de estas variables permite dirigir las intervenciones terapéuticas de acuerdo a las necesidades del paciente.

Una limitación de esta investigación se refiere al hecho de no disponer de mediciones posteriores que nos hubieses permitido conocer la evolución de los pacientes a largo del tratamiento. Futuros trabajos deberán incorporar información longitudinal de las variables estudiadas.

\section{Reconocimientos}

Este estudio ha sido financiado por el proyecto COPERNICO del Plan Nacional sobre Drogas (Convocatoria 2009) y la Red de Trastornos Adictivos (Convocatoria RETICS 2012, Instituto de Salud Carlos III), Grupo Universidad de Granada.

Agradecemos a Miguel Angel Ruiz, Mª́nchez y Manuela Rivero, psicólogos del Centro Provincial de Drogodependencias de Granada, su colaboración en esta investigación.

\section{Conflicto de intereses}

Los autores declaran que no existe ningún conflicto de intereses.

\section{Referencias}

Albein-Urios, N., Martínez-González, J. M., Lozano-Rojas, O. y Verdejo-García, A. (2011). Estudio preliminar para la validación de la versión española del Personality Belief Questionnaire. Trastornos Adictivos, 13, 144-50.

APA, (2002). DSM-IV-TR Manual diagnóstico y estadístico de los trastornos mentales, IV Texto Revisado. Barcelona: Masson.

Beck, A. T. y Beck, J. S. (1991). The Personality Belief Questionnaire. Unpublished assessment instrument. Bala $\mathrm{Cy}$ nwyd, PA: The Beck Institute for Cognitive Therapy and Research.

Becoña, E., Cortés, M., Arias, F., Barreiro, C., Berdullas, J., Iraurgui, I.,...Villanueva, V. (2011). Manual de Adicciones para Psicólogos Especialistas en Psicología Clínica en Formación. Barcelona: Socidrogalcohol.

Bordin, E. (1979). The generalizability of the psychoanalytic concept of the working alliance. Psychoterapy: Theory, Research and Practice, 16, 252-260. doi:10.1037/ h0085885.

Caballero, L. (1993). Determinantes y significado de la retención en programas para drogodependientes. En M. Casas y M. Gossop (Coord), Recaída y prevención de recaídas. Barcelona: Citran.

Caballero, L (2005). Adicción a cocaína: neurología clínica, diagnóstico y tratamiento. Madrid: PNSD.

Daley, D. C y Zuckoff, A (2004). Cómo mejorar el cumplimiento terapéutico en la patología dual y pacientes drogodependientes. Barcelona: Glosa.

Epstein, D. H., Willner-Reid, J., Vahabzadeh, M., Mezghanni, M., Lin, J. L., Preston, K. L. (2009). Real-time electronic diary reports of cue exposure and mood in the hours before cocaine and heroin craving and use. Archives of General Psychiatry, 66, 88-94. doi:10.1001/archgenpsychiatry.2008.509.

Fernández, E., López, A. y Becoña, E. (2010). Trastornos de la Personalidad y abstinencia del consumo de tabaco 
en un tratamiento psicológico para dejar de fumar. Psicothema, 22, 357-362.

First, M., Spitzer, R., Gibbon, M. y Williams, J. (1999). Guía del usuario para la entrevista clínica estructurada para los trastornos de la personalidad del Eje I del DSM-IV (SCID-I). Madrid: Masson.

Forcada, R., Pardo, N. y Bondia, B. (2006). Impulsividad en dependientes de cocaína que abandonan el consumo. Adicciones, 18, 111-118.

Golberg, D. P. y Hiller, V. F. (1979). A scaled version of the General Health Questionaire. Windsor: NFER Publishing Company.

González-Saiz, F. M., Carulla, S., Martínez J. M., López, A., Ruiz, J. y Guerra, D. (1997). Indicador del tratamiento de la adicción a opiáceos. Cádiz: Servicio de Publicaciones de la Universidad de Cádiz.

Hersoug, A. G., Monsen, J. T., Havik, O. E. y Hoglend, P. (2002). Quality of early working alliance in psychotherapy: diagnoses, relationship and intrapsychic variables as predictors. Psychother Psychosom, 71, 18-27. doi: $10.1159 / 000049340$

Higgins, S., Budeney, A., Bickel, W., Foerg, F., Donham, R. y Badger, G. (1994). Incentives improve outcome in outpatient behavioral treatment of cocaine dependence. Archive General Psychiatry, 1, 568-76. doi:10.1001/archpsyc. 1994.03950070060011.

Iraurgi, I., Sanz, M. y Martínez-Pampliega, A. (2004). Funcionamiento familiar y severidad de los problemas asociados a la adicción a drogas en personas que solicitan tratamiento. Adicciones, 16, 185-95.

Jackson, T. R. (2002). Treatment practice and research issues in improving opioid treatment outcomes. Science and Practice Perspectives, 1, 22-28.

Kokkevi, A., Stefanis, N., Anastasopoulou, E. y Kostogianni, C. (1998). Personality disorders in drug abusers. Prevalence and their association with axis I disorders as predictors of treatment retention. Addictive Behaviors. 23, 841-853.

Lambert, M. J., Hansen, N. B. y Finch, A. E. (2001). Patient-focused research: using patient outcome data to enhance treatment effects. Journal of Consulting and Clinical Psychology, 69, 159-72. doi:10.1037/0022-006X.69.2.159.

Lingiardi, V. Filippucci, L. y Baiocoo, R. (2005). Therapeutic Alliance evaluation in personality disorder psychotherapy. Psychotherapy Research, 15, 45-53. doi: 10.1080/10503300512331327047.

Long, C. G., Williams, M. y Hollin, C. R. (1999). Within treatment factors as predictors of drinking outcome following cognitive-behavioral treatment. Addiction Biology, 4, 238-239.

López, A., Becoña, E., LLoves, M. Moneo, A., Viestez, I., Camelo, J.,... Díaz, E. (2007). Evolución del tratamiento y consumo en personas con dependencia de la cocaína a los doce meses en función de la psicopatología asociada y funcionamiento cotidiano. Adicciones, 19, 251-266.

López, A., Becoña, E., Casete, L., Lage, M. Díaz, E., García, J.,... Moneo, A. (2007). Dependencia de la cocaína y trastornos de personalidad. Análisis de su relación en una muestra clínica. Trastornos Adictivos, 9, 215-227.

López-Ibor, J., Pérez Urdániz, A. y Rubio, V. (1996). Examen Internacional de los Trastornos de la Personalidad; Módulo $D S M-I V$. Versión Española. Madrid: Organización Mundial de la Salud.

Lozano, O. M., Rojas, A. J. y Pérez, C. (2006). Construcción de un test para medir la calidad de vida relacionada con la salud especifico para drogodependientes. Sevilla: Junta de Andalucía.

Loranger, A., Lenzenweger, M., Gartner, A., Lehmann, V., Herzig, J., Zammit, G,... Young, R. (1991). Trait State artifacts and the diagnosis of personality disorders. Archives of General Psychiatry, 48, 720-728. doi:10.1001/ archpsyc. 1991.01810320044007.

McMahon, R., Kelley, A. y Kouzekanani, K. (1993). Personality and coping styles in the prediction of dropout from treatment for cocaine abuse. Journal of Personality Assessment, 61, 147-155. doi:10.1207/s15327752jpa6101_11.

Martínez-González J. M., Graña J. y Trujillo H. (2009). Influencia de los trastornos de la personalidad y patrones de consumo en la eficacia de un programa de prevención de recaídas para el tratamiento del alcoholismo. Adicciones, 21, 105-111.

Martínez-González J. M., Graña, J. y Trujillo, H. (2010). La calidad de vida en alcohólicos con trastornos de la personalidad: relación con el ajuste psicológico y el craving. Psicothema, 22, 562-567.

National Institute on Drug Abuse (2002). In drug abuse, gender matters. NIDA notes, 17, 3-5.

Pretzer, J. (1998). Cognitive-behavioral approaches to the treatment of personality disorders. En C. Perris, P.D. McGorry y D. Patric, Cognitive psychoterapy of psicotic and personality disorders. Handbook of theory and practice (pp.269-291). Nueva York: John Wiley and sons.

Pedrero, E. y Puerta, C. (2001). Atención a usuarios de cocaína desde un centro de atención a drogodependencias (CAD-4). Trastornos Adictivos, 3, 11-20.

Perry, J. C. y Bond, M. (2002). Estudios empíricos sobre psicoterapia en trastornos de la personalidad. En J. G. Gunderson y G. O. Gabbard. Psicoterapia de los trastornos de la personalidad (pp.1-35). Barcelona: Ars Médica.

Sánchez-Hervás, E., Secades, R., Santonja, F., Zacarés, F., García-Rodríguez, O., Martín, E.,... García, G. (2010). Abandono del tratamiento en adictos a cocaína. Adicciones, 22, 59-64.

Sánchez-Hervas, E., Tomás, V., Molina, N., Del Olmo, R. y Morales, E. (2002). Procesos de cambio en conducta adictiva. Adicciones, 14, 337-344. 
Sargeant, M., Bornavolova, M., Trotman, A. y Lejnez, C. (2012). Facets of impulsivity in the relationship between antisocial personality and abstinence. Addictive Behaviors, 37, 293-298. doi:10.1016/j.addbeh.2011.11.012.

Sirvent, C. (2009). Fracaso, abandono y cumplimiento terapéutico. Norte de Salud Mental, 33, 51-58.

Sirvent, C. (2010). Predicción del abandono (vs. éxito) terapéutico en adicciones. Presentación del cuestionario VPA30 de variables de abandono y adherencia al tratamiento en adicciones. Interpsiquis: congreso virtual de psiquiatría. $11^{\circ}$ Congreso.

Tyrer, P. y Davidson, K. (2002). Terapia cognitiva en los trastornos de la personalidad. En J.G. Gunderson y G.O. Gabbard. Psicoterapia de los trastornos de la personalidad (pp.131-149). Barcelona: Ars Médica.

Urbanoski, K, Kelly, J., Hoeppner, B. y Slaymaker, V. (2012). The role of therapeutic alliance in substance uso disporder treatment for young adults. Journal of Substance Abuse Treatment, 43, 344-351. doi:10.1016/jjsat.2011.12.013.

Verdejo-García, A., Lozano, O., Moya, M., Alcázar, M.A. y Pérez-García, M. (2010). Psychometric properties of a Spanish version of the UPPS-P impulsive behavior scale: reliability, validity and association with trait and cognitive impulsivity. Journal of Personality Assessment, 92, 70-7. doi:10.1080/00223890903382369.

Vergara-Moragues, E., González-Saiz, F., Lozano, O. y Verdejo-García, A. (2013). Psiquiatric profile of three-month relation in cocaine-dependent patients treated in a therapeutic community. Journal Study Alcohol Drugs, 74, 452-459.

Verheul, R., Van den Bosch, L. y Ball, S. (2007). Abuso de sustancias. En J. M. Oldham, A. E. Skodol y D. Bender, Tratado de los trastornos de la personalidad (pp. 465-478). Madrid: Elsevier Masson.

Whiteside, S. y Lynam, D. (2001). The Five Factor Model and impulsivity: using a structural model of personality to understand impulsivity. Personality and Individual Differences, 30, 669-689. doi: 10.1016/S0191-8869(00)00064-7 